\title{
Biofilms to die for...
}

DOI:

10.1038/nrmicro1684

URLs

Online Links Staphylococcus aureus

http://www.ncbi.nlm. nih.gov/entrez/query. $\mathrm{fcgi}$ ? $\mathrm{db}=$ genomeprj $\& \mathrm{cmd}=$ Retrieve \&do $\mathrm{pt}=$ Overview\&list_ uids $=12304$

...bacterial extracellular genomic DNA is an important structural component of the $S$. aureus biofilm matrix
Many infections caused by Staphylococcus aureus involve the formation of biofilms, communities of differentiated, surface-adhering bacteria surrounded by an extracellular matrix. New research published in Proceedings of the National Academy of Sciences USA reveals that bacterial extracellular genomic DNA is an important structural component of the S. aureus biofilm matrix. Furthermore, these findings also suggest that controlled bacterial programmed cell death and lysis is responsible for the release of genomic DNA.

Previous work by the authors had demonstrated that the cidABC and $\operatorname{lrg} A B$ operons of $S$. aureus regulate cell lysis during the stationary phase of growth and in response to antibiotic exposure. These studies led to the proposal that the cid and lrg operons encode components of a bacterial cell death and lysis process, although a biological role for this system has remained elusive. To explore the biological role of S. aureus cell death and lysis, Ken Bayles and colleagues analysed the effect of a mutation in $\operatorname{cid} A$, the first gene in the cid operon, on biofilm development. Their analysis revealed that by mutating the gene there was a dramatic effect on biofilm morphology and adherence. Biofilms formed by the $\operatorname{cid} A$ mutant had a rougher appearance and demonstrated less adherence to surfaces compared with biofilms produced by the wild type $S$. aureus strain. In addition, propidium iodide staining indicated the presence of an increased number of dead bacterial cells within the biofilm formed by the cidA mutant strain, a finding that was indicative of reduced cell lysis. This observa- tion was confirmed when the authors, using quantitative PCR, detected significantly less genomic DNA in the cidA mutant biofilm. Indeed, exposure of the biofilm formed by the wild-type strain to DNase I treatment had a major impact on the structural integrity of the biofilm. By contrast, using this treatment to reduce the amount of extracellular genomic DNA within the cidA mutant biofilm resulted in only a modest effect on biofilm formation, a finding consistent with the reduced amounts of DNA in this biofilm.

Collectively, these observations support the assertion that genomic DNA released by $S$. aureus cells is an important and necessary structural

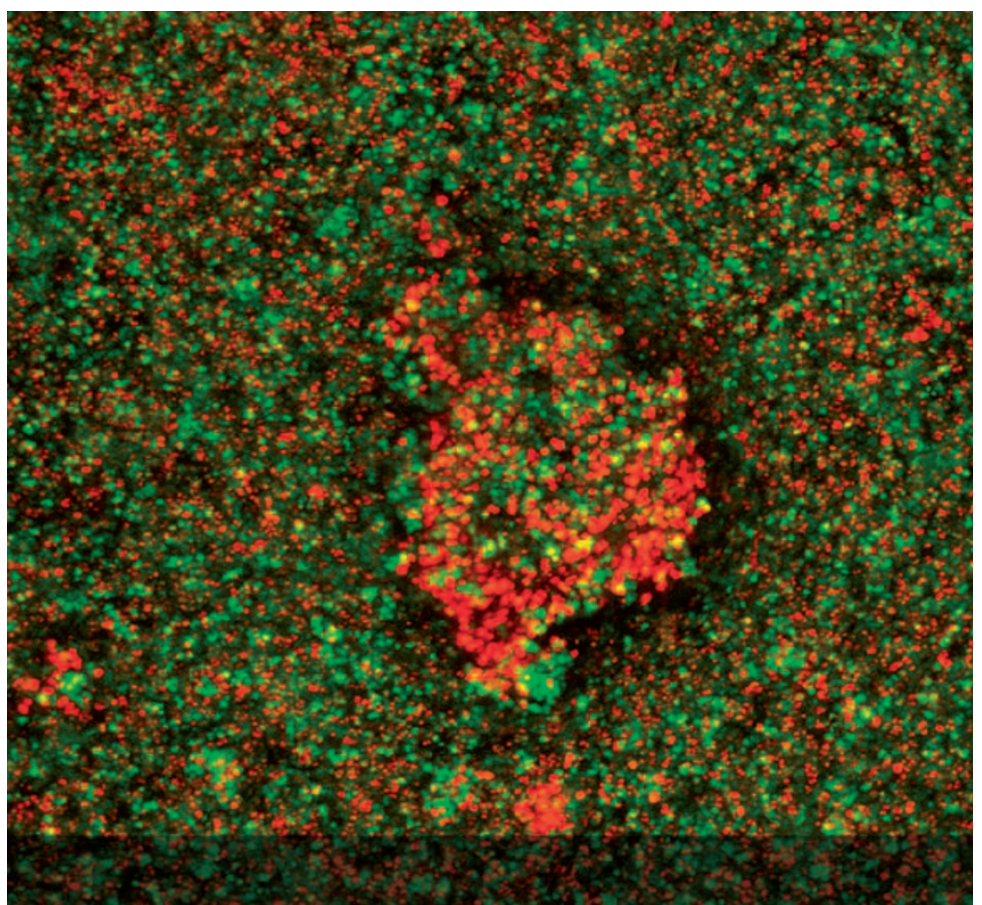

A confocal image ( $400 \times$ magnification) showing a mixed population of live (green) and dead (red) Staphylococcus aureus cells within a 3-day-old biofilm. Image provided by Ethan Mann, University of Nebraska Medical Center, Nebraska, USA component of the $S$. aureus biofilm matrix and that cidA-controlled bacterial cell death and lysis has a significant role in this process. As homologues of $c i d / l$ rg genes are present in a wide range of bacteria - Gram-positive and Gram-negative - future work will assess the involvement of these conserved genes in biofilm formation and, intriguingly, the relationships between this system and the role of programmed cell death in more complex multicellular organisms.

David O’Connell

ORIGINAL RESEARCH PAPER Rice, K. C. et al. The cidA murein hydrolase regulator contributes to DNA release and biofilm development in Staphylococcus aureus. Proc. Natl Acad. Sci. USA 23 Apr 2007 (doi:10.1073/pnas.0610226104) 